\title{
Cauchemar ou réalité?
}

\section{René Bloch}

Dr méd., spécialiste en psychiatrie et psychothérapie, membre de la FMH

Jusqu'au milieu du $\mathrm{XX}^{\mathrm{e}}$ siècle, on ne pouvait guère prétendre que l'humanité courait à sa perte en raison des effets secondaires négatifs du progrès technique. Seul un petit nombre de scientifiques avait compris que le développement technologique et l'expansion économique menaçaient l'avenir de la société humaine. Les avantages économiques de ces deux tendances étaient

\section{Les mises en garde n'ont pas été entendues et se sont noyées dans l'ivresse de la croissance économique.}

si évidents qu'on en a oublié que la croissance atteindrait un jour ses limites et que les ressources et réserves énergétiques de la planète ne sont pas infinies. L'après-guerre a été marqué par une euphorie de la reconstruction et dans les décennies qui ont suivi, on a tenté d'étendre la nouvelle opulence à toute la planète à travers la mondialisation. Les mises en garde n'ont pas été entendues et se sont noyées dans l'ivresse de la croissance économique.

Aujourd'hui, on croit à un cauchemar lorsque les médias alertent sur le possible effondrement de l'écosystème de la planète, avec toutes les conséquences qu'il aurait sur les moyens de subsistance de l'humanité. Du fait de la surexploitation de la planète, de sa pollution et de son réchauffement, les équilibres naturels ont déjà été gravement perturbés et l'éventualité d'une rupture s'est rapprochée.

Depuis une dizaine d'années, une prise de conscience des risques s'est produite et de vastes mesures ont été lancées à l'échelle mondiale pour éviter une nouvelle détérioration des biotopes aux répercussions catastrophiques. Les mesures à prendre exigent la coopération de tous les membres de la communauté internationale. Un objectif ambitieux, dont la réalisation est peu probable. Tout récemment, le Groupe d'experts intergouvernemental sur l'évolution du climat a lancé un appel à l'union, pour limiter le réchauffement de la terre à 1,5 degré. Il a souligné que le moment était venu d'agir immédiatement et résolument et que l'heure n'était plus aux déclarations de bonnes intentions. Au vu des dérèglements climatiques déjà intervenus, ses avertissements ne peuvent être ignorés. Les réactions politiques n'en sont que plus sidérantes. Certes, les gouvernements veulent s'engager en faveur des objectifs climatiques définis, mais émettent en même temps d'innombrables réserves à l'encontre de mesures immédiates et drastiques sur le terrain. La liberté d'action se heurte à la crainte d'un ralentissement de la croissance économique et du chômage. Les défis posés par la situation actuelle seront difficiles à résoudre sans bouleversements sociaux majeurs. Il est vrai que cette situation de crise est due au fait que l'on a attendu trop longtemps pour remédier au dérapage de la relation entre l'être humain et la nature. Si l'on ne parvient pas aujourd'hui à évaluer correctement les priorités et à placer en tête de liste la sauvegarde de la nature et des équilibres écologiques, il se pourrait tout à fait qu'il soit trop tard demain et que les écosystèmes s'effondrent, entraînant la disparition de la plupart des êtres vivants. L'humanité dans son ensemble est aujourd'hui confrontée à l'une des décisions les plus difficiles de son histoire: soit prendre des mesures immédiates dans le monde entier pour éviter la poursuite de la destruction de l'environnement naturel en espérant qu'il soit encore temps d'arrêter une évolution chaotique, soit tenter de maintenir la prospérité atteinte pendant quelques décennies, sans tenir compte des conséquences sur l'avenir.

L'humanité dans son ensemble est aujourd'hui confrontée à l'une des décisions les plus difficiles de son histoire.

En parallèle de la lutte contre le changement climatique anthropique, il faut s'efforcer de maîtriser ses effets sur la santé. Le dérèglement climatique a de vastes conséquences sur la santé de certains individus et de la population dans son ensemble. Sous l'influence du stress thermique ou des polluants atmosphériques, le taux de mortalité de plusieurs maladies a augmenté. La recherche concernant les effets du changement climatique sur la santé est relativement avancée par rapport aux études sur les moyens de le contrer, car certains de ces moyens semblent difficiles à appliquer et tabous. 